\title{
Response style corrected market segmentation for ordinal data
}

\author{
Bettina Grün $^{1}$ - Sara Dolnicar ${ }^{2}$
}

Published online: 18 June 2015

(C) The Author(s) 2015. This article is published with open access at Springerlink.com

\begin{abstract}
Survey data collected for market segmentation studies is typically ordinal in nature. As such, it is susceptible to response styles. Ignoring response styles can lead to market segments which do not differ in beliefs, but merely in how segment members use survey answer options and which possibly occur in addition to the belief segments. We propose a finite mixture model which simultaneously segments and corrects for response styles, permits heterogeneity in both beliefs and response styles, accommodates a range of different response styles, does not impose a certain relationship between the response style and belief segments, and is suitable for ordinal data. The performance of the model is tested using both artificial and empirical survey data.
\end{abstract}

Keywords Market segmentation $\cdot$ Ordinal data $\cdot$ Response style $\cdot$ Heterogeneity

\section{Introduction}

Response styles are consistent tendencies displayed by survey respondents to "respond to a range of questionnaire items on some basis other than the specific item content" (Paulhus 1991, p.17), such as the tendency to tick extreme response options (extreme response style) or the tendency to agree (acquiescence response style; Baumgartner and Steenkamp 2001).

Response styles are problematic in survey research. Once data has been collected, it is difficult to separate respondents' beliefs from their response styles. The inseparability of beliefs and response styles can lead to the misinterpretation of response styles as content. This occurs, for example, when correlation-based methods, such as factor or

Bettina Grün

bettina.gruen@jku.at

Sara Dolnicar

s.dolnicar@uq.edu.au

1 Johannes Kepler University Linz, Altenbergerstraße 69, 4040 Linz, Austria

2 University of Queensland, St Lucia, Brisbane 4072, Australia 
regression analyses, are employed because extreme response styles skew the frequency distribution toward the end points thus increasing standard deviation and decreasing correlation (Heide and Gronhaug 1992). Response styles also affect covariance-based analyses: Rossi et al. (2001) show that correlation inferences are different once adjustments are made for the discreteness of the data and differences in scale usage. Finally, and most importantly, in the context of market segmentation, algorithms which are based on distance measures (Greenleaf 1992a), such as cluster analysis, detect response style segments. Such segments might often occur in addition and be easily misinterpreted by users as meaningful segments.

The presence of response styles in survey data can for example be checked based on specific measures (e.g., see Baumgartner and Steenkamp 2001). If the presence of response styles is confirmed, suggestions on how to remove response style bias retrospectively include the following:

(1) Using additional questions to identify the nature of each survey participant's response style for the purpose of correction (Greenleaf 1992b).

(2) Standardizing responses. Note, however, that Fischer (2004) warns against unwanted effects of the standardization, such as the occurrence of spurious method factors in factor analysis, and the complication of interpretation.

(3) Robustness-based approaches correct data using a number of suitable methods and then assess the effect on the results after data analysis (Dolnicar and Grün 2007).

(4) Integrated models of simultaneous data analysis and response style correction (Rossi et al. 2001; Johnson 2003; Javaras and Ripley 2007). These models assume that responses on an ordinal scale result from the mapping of a latent continuous variable, and that respondents differ in response styles, but are homogeneous with respect to their beliefs. The latter assumption contradicts the basic assumption of market segmentation that groups of consumers - market segments - differ in their beliefs. Later, another model was proposed which simultaneously segments and corrects for response styles while allowing segments to differ in beliefs (Van Rosmalen et al. 2010). Its use is limited, however, to nominal data.

We propose a finite mixture model that identifies belief segments and response styles simultaneously, can accommodate different response styles, and can handle ordinal data. The proposed method avoids two problems: (1) the incorrect omission of market segments because response style correction before segmentation analysis removed heterogeneity in beliefs and (2) the incorrect creation of segments based on heterogeneity in response styles only when response styles are not corrected for.

\section{Model}

The model assumes that the observed answer $z_{i j}$ of respondent $i$ for question $j$ is constructed by the respondent by mapping the value of a latent continuous variable $y_{i j}$ representing the true belief onto the ordinal scale using an individual mapping function which is identical across survey questions (cf. Bradlow and Zaslavsksy 1999). This assumption is crucial to being able to separate the effect of the response 
styles from the content information. The assumption is reasonable given that people display response biases consistently across time and situations (Paulhus 1991). The relationship between the observed answer and the latent variable is given by

$$
z_{i j}=k \Leftrightarrow c_{i(k-1)}<y_{i j} \leq c_{i k}
$$

The observed answers $z_{i j}$ take values in $\{1, \ldots, K\}$ if the measurement is on a $K$-point scale. For the individual thresholds $C_{i}=\left(c_{i k}\right)_{k=0, \ldots, K}$, the following holds

$$
-\infty=c_{i 0}<c_{i 1}<\ldots<c_{i(K-1)}<c_{i K}=\infty .
$$

The specification with individual thresholds - which reflect the respondent's response style - offers maximum flexibility in capturing different kinds of response biases.

The value of the underlying continuous variable is assumed to be given by

$$
y_{i j}=x_{i j}^{T} \beta_{i}+\varepsilon_{i j}
$$

where $\varepsilon_{i j}$ is independently identically distributed $N(0,1)$. This assumes that the observed item and respondent characteristics $x_{i j}$ determine the mean of the continuous variable depending on the respondent-specific regression coefficients $\beta_{i}$. This implies that respondents may vary in the mean values of the underlying continuous variable due to observed variables $x_{i j}$; unobserved heterogeneity between respondents is captured by the respondent-specific regression coefficients $\beta_{i}$. The proposed approach - similarly to the conditional independence assumption in latent class analysis - assumes that the evaluations are independent given the observed variables $x_{i j}$ and the regression coefficients $\beta_{i}$, i.e., there is no remaining dependency structure between item evaluations. This assumption has the computational advantage that only univariate problems for each of the items need to be solved instead of a multivariate probit model. Furthermore, Hennig and Liao (2013) point out that the assumption of conditional independence in finite mixture models makes the interpretation of segmentation analyses easier.

To ensure identifiability of the model, the thresholds and regression coefficients cannot both be freely selected. One possibility is to anchor the latent variable $y_{i j}$ at a convenient point in order to allow easy interpretation of the parameters (for example, the average evaluation could be assumed to be zero). This model specification allows for respondent-specific effects with respect to response styles (as indicated by the thresholds $C_{i}$ ) and measured content (as indicated by the regression coefficients $\beta_{i}$ ).

Estimating these effects on an individual basis is generally not possible. Restrictions can be imposed to simplify the model and reduce its flexibility. Suitable model simplifications will depend on the assumed nature of heterogeneity between respondents, i.e., discrete or continuous (for a discussion, see Wedel et al. 1999). In the present study, discrete segments are assumed to be present with respect to beliefs held and response styles displayed, leading to both belief and response style segments (for a similar approach, see Bodapati 2008). Because belonging to a certain response style segment is not assumed to be associated with belonging to a certain belief segment, a joint segmentation approach (Ramaswamy et al. 1996) is used. 
Assuming that there are $R$ response style segments and $B$ belief segments the model is given by

$$
P\left(z_{i j}=k \mid x_{i j}, \theta\right)=\sum_{r=1}^{R} \sum_{b=1}^{B} \pi_{r b} P\left(z_{i j}=k \mid x_{i j}, C_{r}, \beta_{b}\right)
$$

with $\pi_{r b} \geq 0$ for all $r$ and $b$ and $\sum_{r=1}^{R} \sum_{b=1}^{B} \pi_{r b}=1$ and $\theta=(\pi, C, \beta)$, where $\pi=\left(\pi_{r b}\right)_{r=1, \ldots, R ; b=}$ $1, \ldots, B, C=\left(C_{r}\right)_{r=1, \ldots, R}$, and $\beta=\left(\beta_{b}\right)_{b=1, \ldots, B}$. In addition, the probabilities are given by

$$
P\left(z_{i j}=k \mid x_{i j}, C_{r}, \beta_{b}\right)=\Phi\left(c_{r k}-x_{i j}^{T} \beta_{b}\right)-\Phi\left(c_{r(k-1)}-x_{i j}^{T} \beta_{b}\right)
$$

where $\Phi$ is the cumulative standard normal distribution function.

This model is a finite mixture of regressions (see for example Wedel and Kamakura 2000; Becker et al. 2014) with segments for response styles and beliefs. These two types of segments are not assumed to occur independently but can be arbitrarily combined and the resulting size of the combination is indicated by $\pi_{r b}$. Assuming that a certain response style can be associated with several belief segments or that different response styles occur together with the same belief segment, this model formulation is more parsimonious than a model where each segment represents a separate belief and response style segment. It also allows the response style distribution to vary over belief segments, and therefore, the co-occurrence of a certain response style and a certain belief is not necessarily independent. The model is estimated with the EM algorithm (see Appendix).

\section{Simulation study}

The proposed modeling approach is validated using simulated data. Data from four different scenarios are sampled:

SCENARIO \#1 Respondents are homogeneous in terms of both their beliefs and response styles (no segments).

SCENARIO \#2 Respondents are heterogeneous in terms of their beliefs but homogeneous in terms of response styles (belief segments only).

SCENARIO \#3 Respondents are homogeneous in terms of beliefs and heterogeneous in terms of response styles (response style segments only).

SCENARIO \#4 Respondents are heterogeneous both in terms of beliefs and response styles (response style and belief segments), and there is no association between group membership for response styles and belief segments.

In addition, segments — when present — are either (a) of equal size or (b) of unequal size containing one small segment with $10 \%$ of the respondents. One hundred datasets are generated for each scenario in combination with the different segment sizes. Each 
data set contains 420 respondents who responded to ten questions using a 7-point scale. Response style segments - when present - either (1) tend to use the middle of the scale and provide symmetric responses (i.e., thresholds are generated using quantiles from the standard normal distribution for eight equidistant values from 0 to 1 multiplied by two), (2) display an extreme response style (i.e., the thresholds of segment 1 are divided by two), or (3) display a combined acquiescence and extreme response style (i.e., the thresholds of segment 1 are divided by two and then shifted to the left by one).

Belief segments - when present — differ as follows: belief segment 1 has a value of 1 for the first three beliefs, 0 for the next four, and -1 for the last three. Belief segment 2 has all values multiplied by -1 , and belief segment 3 has a value of 0 for the first two beliefs, a value of 1 for the next three, a value of -1 for the next three, and 0 for the last two beliefs. Belief segment 4 has the opposite values than belief segment 3. If no segments are present, all respondents have the thresholds or the mean evaluations of the latent variables of segment 1 . Note that the segmentation task is rather easy if only one source of heterogeneity is present and segments are of equal size, with the task being more difficult in the other settings.

The model is fitted to each of the 700 datasets. The number of response style segments is varied from 1 to 4 , and the number of belief segments from 1 to 5 and all combinations are fitted. The best model is selected using the BIC. The results of the simulation study are summarized in Table 1. The percentage of the correctly selected number of response style segments and belief segments using the BIC is determined. For the first three scenarios (no heterogeneity, belief segments only, response style segments only) with equal segment size, the correct model is selected for all 100 replications. Only for the scenario where both belief and response style segments exist, the correct model selection rate drops to $97 \%$ for the belief segments. If a small segment is present, the performance of the BIC deteriorates considerably, especially when both response style and belief segments are present. BIC, thus, appears not to be the optimal criterion for niche segment detection as it aims at selecting a model which best fits the dataset as a whole.

Table 1 Model fit for 100 replications for four scenarios with equal and unequal segment sizes

\begin{tabular}{|c|c|c|c|c|}
\hline & \multicolumn{2}{|c|}{ Correct model selection } & \multicolumn{2}{|c|}{$\begin{array}{l}\text { Mean (std. dev) of correct } \\
\text { classification rate }\end{array}$} \\
\hline & Response style & Belief & Response style & Belief \\
\hline No segments & $100 \%$ & $100 \%$ & $1.00(0.00)$ & $1.00(0.00)$ \\
\hline \multicolumn{5}{|l|}{ Equal segment sizes } \\
\hline Only belief segments & $100 \%$ & $100 \%$ & $0.89(0.01)$ & $1.00(0.00)$ \\
\hline Only response style segments & $100 \%$ & $100 \%$ & $1.00(0.00)$ & $0.88(0.02)$ \\
\hline Opinion and response style segments & $100 \%$ & $97 \%$ & $0.88(0.05)$ & $0.86(0.04)$ \\
\hline \multicolumn{5}{|l|}{ Unequal segment sizes } \\
\hline Only belief segments & $100 \%$ & $72 \%$ & $0.88(0.03)$ & $1.00(0.00)$ \\
\hline Only response style segments & $89 \%$ & $100 \%$ & $1.00(0.00)$ & $0.87(0.03)$ \\
\hline Opinion and response style segments & $56 \%$ & $24 \%$ & $0.86(0.04)$ & $0.83(0.04)$ \\
\hline
\end{tabular}


In the following, we compare the models selected using the BIC with respect to their ability to cluster the respondents into response style and belief segments. Cluster labels are assigned to both response style and belief segments according to the maximum a posteriori probabilities by maximizing the correct classification rate for each of the segmentations separately. The correct classification rate is an important criterion because the correct cluster structure is also required to identify suitable covariates which are correlated with clusters and can be used for marketing action. The mean values of the correct classification rate as well as the standard deviations are given in Table 1. Clearly, the classification rate is perfect if respondents are homogeneous. If there is only one source of heterogeneity, about $90 \%$ of respondents are correctly classified for equal segment sizes. This percentage drops only slightly when one small segment is present.

If both sources of heterogeneity are present, the correct classification rate is similarly high, indicating that the approach is capable of retaining the correct classifications. The correct classification rates for the belief segments were also calculated if no response style segments are included in the estimated model. If response style and belief segments are present, the correct classification rate of the belief segments then drops from 86 to $62 \%$ for equal segment sizes and from 83 to $76 \%$ for unequal segment sizes.

To investigate model performance in a more difficult setting, we drew 100 datasets from the model estimated using empirical survey data (see Sect. 4) where four belief and five response style segments are detected. Models were fitted using the artificial data, and BIC was used for model selection. In all cases, four belief segments were selected. The selected number of response style segments was correct in $95 \%$ of the cases and underestimated by one in $5 \%$. If response styles are ignored, the correct number of belief segments is selected in only $80 \%$ of cases (4\% underestimation, $16 \%$ overestimation).

\section{Application: evaluation of fast food restaurants}

Brand image evaluations of a fast food restaurant are analyzed. Seven hundred and fifteen Australian adult respondents evaluated "McDonalds" using 10 attributes (cheap/ expensive, convenient/inconvenient, disgusting/delicious, fast/slow, fattening/not fattening, greasy/not greasy, healthy/unhealthy, spicy/mild, tasty/bland, yummy/yuk) on a 7-point fully verbally labeled answer scale. For identifiability, it is assumed that the sum of the regression coefficients for the 10 attributes is zero.

Different finite mixture models were fitted with the EM algorithm where the number of response styles and belief segments was varied from 1 to 9 and each combination thereof as long as the total number of segments, which corresponds to the number of response style segments times the number of belief segments, is smaller or equal to 36 .

If the same thresholds are imposed for all respondents, this leads to a model where differences between respondents are assumed to only occur due to differences in beliefs and differences in response styles - if they existed - would also need to be captured by the belief segments. In this case, a model with six segments is selected using the BIC where each segment should correspond to a different belief segment. The patterns of the belief segments are shown at the top of Fig. 1. Because the range of the latent variable 
Without response style segments

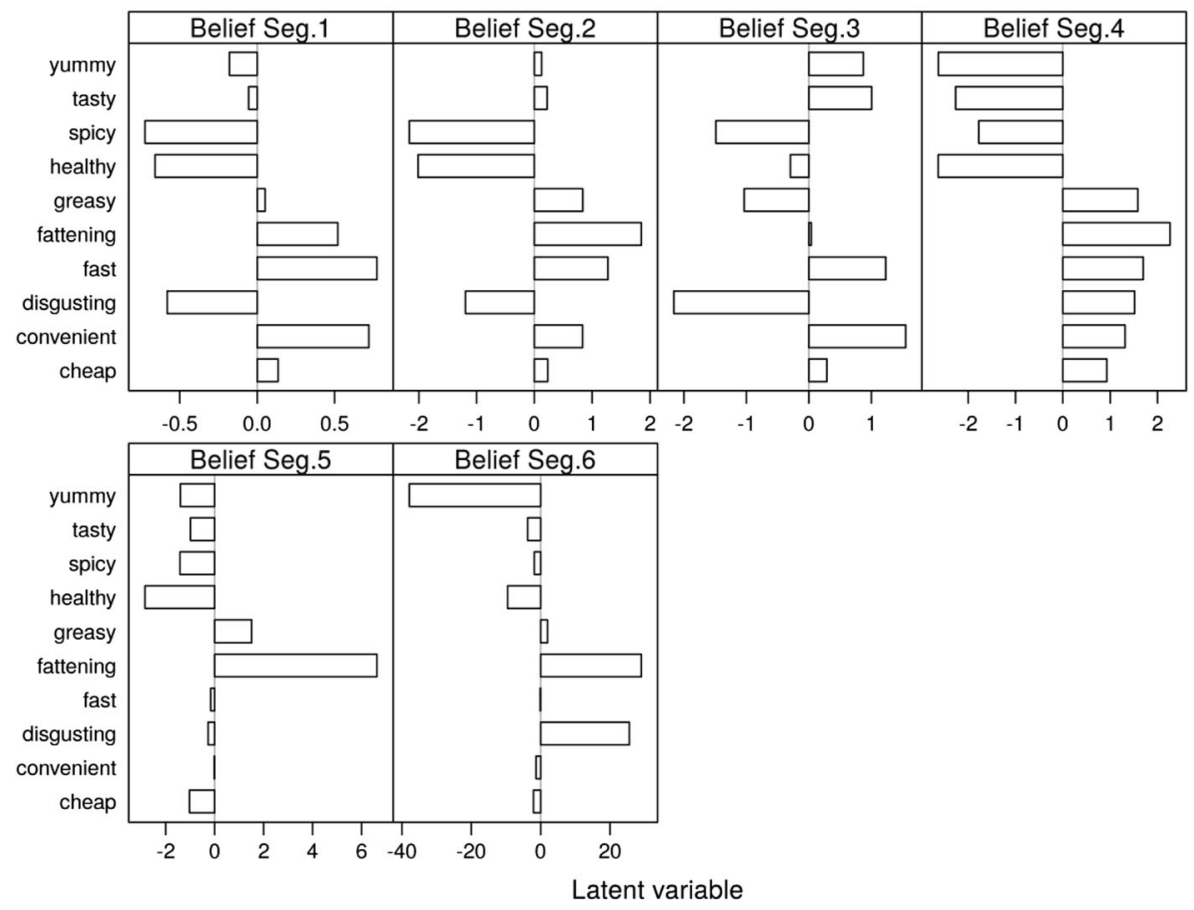

With response style segments

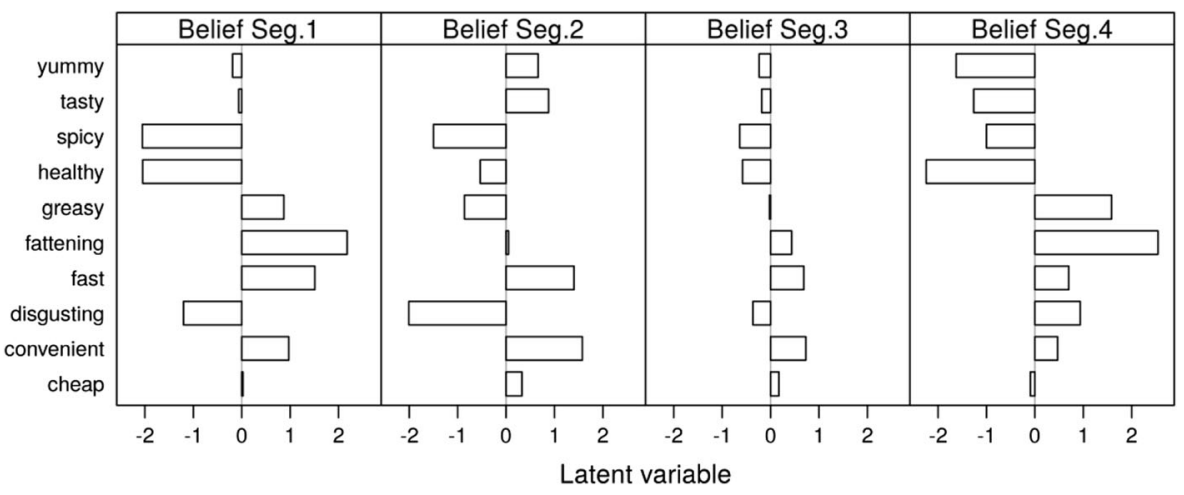

Fig. 1 Estimated latent agreement levels with the 10 attributes for belief segments

varies strongly across segments, a different scale for the $x$-axis is used for each segment. The segments are ordered by size from largest to smallest; the corresponding segment sizes are 47, 20,17, 7,6, and $3 \%$. The estimated thresholds are given at the top of Fig. 2; they indicate that the latent variable needs to be positive to elicit agreement, while the middle point is ticked if the latent variable is negative, and that the thresholds are not evenly spaced.

The belief patterns shown at the top of Fig. 1 indicate that members of segment 1 do not like the food at McDonalds and think it is fattening, but acknowledge that eating at 


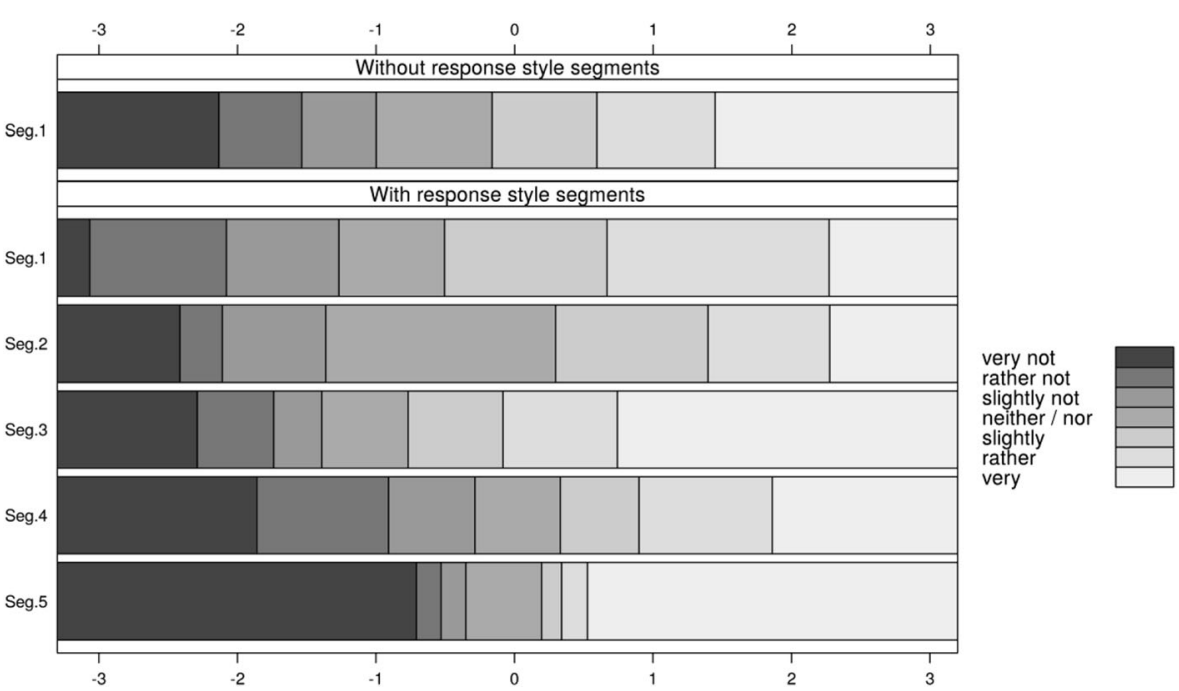

Fig. 2 Estimated thresholds for the mapping of the latent continuous response to the ordinal observed response for the best solution without and with response style segments

McDonalds is fast and convenient. Segment 2 has a similar perception of McDonalds but is more extreme in their evaluation than segment 1 and also more pronounced in perceiving McDonalds as greasy. Segment 3 consists of McDonald fans: they think the food is yummy and tasty, neither greasy nor disgusting and that eating there is fast and convenient. Segments 4 and 5 consist of respondents who do not disagree with the statement that McDonalds is disgusting and think the food is not yummy and fattening, thus viewing McDonalds in the least favorable way. Segment 6 has extreme views in perceiving McDonalds as not yummy, but as fattening and disgusting. This analysis indicates that - while the model differentiated between belief segments - differences between respondents with respect to response styles and beliefs are confounded. For example, segments 1 and 2 have a similar pattern, but a comparison of the $x$-axis reveals that members of segment 1 display a mild response style.

If response styles and belief segments are fitted simultaneously, the best fitting model according to the BIC suggests extracting four belief segments and five response style segments. By accounting for response styles, belief heterogeneity between respondents could be captured in fewer segments (see bottom of Fig. 1; segment sizes 29, 29, 26, and $16 \%$ ). Here, all panels use the same scaling for the $x$-axis. Members of segment 1 think that it is a fattening, but fast and convenient eating option. Members of segment 2 are McDonalds fans who think that the food is yummy and tasty, not greasy and not disgusting and McDonalds is fast and convenient. Segment 3 does not have a strong profile and differs most from segment 1 with respect to the attribute greasy. Segment 4 consists of respondents with a very unfavorable view of McDonalds, who even agree that McDonalds is disgusting.

A comparison of the belief segments identified indicates that the results of the two approaches differ substantially. If response styles are accounted for, four segments of similar size emerge. If response styles are ignored, one large segment containing nearly half of the respondents emerges along with three very small segments, each containing 
fewer than $10 \%$ of respondents. Yet, the key perceptions of McDonalds - loving it, considering it as convenient option, being rather indifferent and disliking it - are discernible in both solutions. The solution without response style segments, however, contains additional spurious solutions which complicate interpretation.

The estimated thresholds for the five response style segments are given at the bottom of Fig. 2: segments differ in their extreme and acquiescence response style. The extreme response style leads to thresholds being less dispersed because the latent continuous variable needs to be less extreme to elicit an extreme answer on the ordinal scale. The acquiescence response style manifests itself by shifting thresholds to the left. Segment 5 displays the highest level of extreme responding, segments 3 and 4 are also susceptible to it, but differ in the extent of their acquiescence response style with segment 3 tending to agree more. Members of segment 1 tend to avoid the extreme answer categories altogether; members of segment 2 only rarely use them.

The relationship between response style segments and belief segments is investigated by assigning each respondent to one response style and one belief segment according to the maximum a posteriori probabilities. The cross-tabulation of these memberships is given in Table 2 .

As can be seen, these two segmentations are not independent (Cramer's $V=$ 0.45 ). The first belief segment coincides primarily with response style segments 2 and 4, while belief segment 2 coincides primarily with response style segments 1 and 3 . Belief segment 3 co-occurs mostly with response style segment 2 , and belief segment 4 with response style segment 4 . This indicates the advantage of a joint segmentation: the assumption that the response style distribution is the same for each belief segment cannot be confirmed. It is this assumption that is implied when assuming independence of the two segmentations. Also, the assumption that only one response style segment is associated with one belief segment cannot be confirmed, rendering the joint segmentation more parsimonious and easier to interpret than a specification where for each segment, the belief and response style are determined separately.

Table 2 Cross-tabulation of belief segment and response style memberships

\begin{tabular}{|c|c|c|c|c|c|c|}
\hline \multirow[t]{2}{*}{ Belief segments } & \multicolumn{6}{|c|}{ Response style segments } \\
\hline & 1 & 2 & 3 & 4 & 5 & Total \\
\hline \multirow[t]{2}{*}{1} & 17 & 57 & 4 & 67 & 4 & 149 \\
\hline & $11 \%$ & $38 \%$ & $3 \%$ & $45 \%$ & $3 \%$ & $100 \%$ \\
\hline \multirow[t]{2}{*}{2} & 108 & 2 & 84 & 30 & 12 & 236 \\
\hline & $46 \%$ & $1 \%$ & $36 \%$ & $13 \%$ & $5 \%$ & $100 \%$ \\
\hline \multirow[t]{2}{*}{3} & 37 & 111 & 57 & 0 & 46 & 251 \\
\hline & $15 \%$ & $44 \%$ & $23 \%$ & $0 \%$ & $18 \%$ & $100 \%$ \\
\hline \multirow[t]{2}{*}{4} & 1 & 12 & 17 & 45 & 4 & 79 \\
\hline & $1 \%$ & $15 \%$ & $22 \%$ & $57 \%$ & $5 \%$ & $100 \%$ \\
\hline Total & 163 & 182 & 162 & 142 & 66 & 715 \\
\hline
\end{tabular}




\section{Summary}

Typical survey data used in market segmentation studies is ordinal in nature. Such data is prone to contamination by response styles. Not correcting for response styles can lead to incorrectly identifying belief segments which do not actually differ in beliefs.

The proposed method is able to distinguish between data situations where no segments exist, only belief segments exist, only response style segments exist and where both belief and response style segments exist. Also, simultaneously accounting for response style and belief segments leads to improved recovery of the belief segments which is essential for developing targeted marketing action.

It can be concluded that the proposed model represents a useful way for market segmentation researchers to improve their analyses, by accounting for response styles while simultaneously deriving the market segments. The proposed model can also easily be extended to include covariates to characterize and profile the response style and belief segments by using concomitant variable models to model the component memberships (Wedel 2002; Wedel and DeSarbo 2002). This would make it possible to investigate which response style segments are related to which sociodemographic characteristics and to determine the respondent composition of the belief segments.

In the proposed model, response style and belief segments are neither assumed to be independent nor are they assumed to coincide. Rather, the combination of the two kinds of segments is flexibly estimated. If results indicate that the two types of segments are either independent or aligned, this restriction can easily be added to the model and this alternative model fitted by suitably modifying the EM algorithm.

The proposed model can easily be extended for use with data of mixed modality: each of the scales then forms a different block with its own thresholds. The conditional independence assumption given segment membership implies that each of these sets of thresholds can be separately determined in the M-step. However, no link between these thresholds is imposed during estimation, i.e., it is not ensured that the estimated thresholds correspond to similar response styles. This flexibility comes at a cost: the approach is only feasible if a small number of different scales is present in the data.

A limitation of the approach is that it needs to be assumed that the overall tendency respondents display when ticking answer categories is primarily related to the response style and not the item content. If violated, the identified response styles may also reflect the overall attitude toward the measured content. The belief segments still offer insights on the differences in measured content. The model then thus disentangles an overall attitude from the relations between item evaluations.

Acknowledgments We thank the Australian Research Council (ARC) and the Austrian Science Fund (FWF) for contributing to the funding of this study through grant DP110101347 and Elise-Richter grant V170-N18. We thank Dominik Ernst for feedback on previous versions of the manuscript.

Open Access This article is distributed under the terms of the Creative Commons Attribution 4.0 International License (http://creativecommons.org/licenses/by/4.0/), which permits unrestricted use, distribution, and reproduction in any medium, provided you give appropriate credit to the original author(s) and the source, provide a link to the Creative Commons license, and indicate if changes were made. 


\section{Appendix: model estimation}

Model estimation of finite mixtures is possible using the expectation-maximization (EM) algorithm (Dempster et al. 1977) within a maximum likelihood framework. Assuming the unobserved segment memberships to be known and given by $w_{i 1}$ and $w_{i 2}$ for respondent $i$ to indicate the response style segment and the belief segment, the complete-data $\log$-likelihood for $n$ respondents is given by

$$
\log L(\theta \mid x, z, w)=\sum_{i=1}^{n}\left[\log \left(\pi_{w_{i 1} w_{i 2}}\right)+\sum_{j=1}^{J} \log \left(P\left(z_{i j}=k \mid x_{i j}, C_{w_{i 1}}, \beta_{w_{i 2}}\right)\right)\right],
$$

where $z=\left(z_{i j}\right)_{i j}, w=\left(w_{i 1}, w_{i 2}\right)_{i}$, and $x=\left(x_{i j}\right)_{i j}$.

To avoid degenerate solutions where thresholds of adjoining values are extremely close, a penalized log-likelihood approach can be pursued. In this case, a penalty is added which is determined as the sum of squared differences of induced proportions for a standard normal distribution of adjoining categories in order to favor solutions where equal probabilities are assigned to all categories. Similar penalties are used to estimate smooth functions for generalized additive models where the differences of coefficients are penalized (Eilers and Marx 1996). In the simulation study and the application, we weight this penalty with 100 in order to reflect the prior belief that differences which are larger than 0.2 are extremely unlikely.

The EM algorithm iterates between an E- and an M-step. In the E-step, the expectation of the complete-data log-likelihood is determined given the data and current parameter estimates for $\theta$. For the proposed model, the E-step consists of determining the a posteriori probabilities of the respondents to be from response style segment $r$ and belief segment $b$ :

$$
\hat{p}_{i, r b}=P\left(w_{i 1}=r, w_{i 2}=b \mid x, z, \theta\right) \propto \pi_{r b}\left[\prod_{j=1}^{J} P\left(z_{i j}=k \mid x_{i j}, C_{r}, \beta_{b}\right)\right] .
$$

In an ordinary EM algorithm, the M-step would consist of maximizing the expected complete-data log-likelihood with respect to the parameters $\theta$. Determining the segment weights, $\pi_{r b}$ is straightforward using

$$
\hat{\pi}_{r b}=\frac{1}{n} \sum_{i=1}^{n} \hat{p}_{i, r b} .
$$

In addition, the segment-specific parameters $C$ and $\beta$ need to be determined. For the proposed mixture model, this part of the M-step is challenging. However, the expectation-conditional-maximization (ECM) algorithm (Meng and Rubin 1993) can be used to exploit the natural partition of the segment-specific parameters into two groups. The original M-step of the EM algorithm is replaced with two CM-steps: (1) the response style segment parameters are determined given the expected completedata log-likelihood and all other current parameter estimates and (2) the belief segment parameters are determined given the expected complete-data log-likelihood and all 
other current parameter estimates. Estimation of the segment weights is done independently of the other parameter estimates given the a posteriori probabilities.

A general purpose optimizer allowing for constraints can be used for the two CM-steps to determine new estimates for the thresholds as well as the regression coefficients. For estimating the thresholds, it is convenient to reparameterize them to ensure they are an ascending sequence. The reparameterization leads to parameters which consist of the smallest threshold (between the first and second answer category) and differences between thresholds. For optimizing the (penalized) complete-data log-likelihood, all but the first parameter are then restricted to be positive. We employ a general purpose optimizer using a quasi-Newton method with box constraints as proposed in Byrd et al. (1995).

Convergence of the EM algorithm is ensured for bounded likelihoods. However, the EM algorithm does not necessarily converge to the global maximum. To increase the chance to detect the global optimum, the initialization strategy proposed by Scharl et al. (2010) is applied. In the simulation study and the empirical application, the following setting is used: the number of random initializations is set to 5 , the loose convergence criterion to $\varepsilon=10^{-4}$ and the stringent convergence criterion to $\varepsilon=10^{-8}$. Model selection is performed by fitting different models with all combinations of different number of response style and belief segments and choosing the best model according to the minimum Bayesian information criterion (BIC; Fraley and Raftery 2002).

\section{References}

Baumgartner, H., \& Steenkamp, J.-B. E. M. (2001). Response styles in marketing research: a cross-national investigation. Journal of Marketing Research, 38(2), 143-156.

Becker, J.-M., Ringle, C. M., Sarstedt, M., \& Völckner, F. (2014). How collinearity affects mixture regression results. Marketing Letters. doi:10.1007/s11002-014-9299-9.

Bodapati, A. V. (2008). Recommendation system with purchase data. Journal of Marketing Research, 45(1), 77-93.

Bradlow, E. T., \& Zaslavsky, A. M. (1999). A hierarchical latent variable model for ordinal data from a customer satisfaction survey with "no answer" responses. Journal of the American Statistical Association, 94(445), 43-52.

Byrd, R. H., Lu, P., Nocedal, J., \& Ciyou, Z. (1995). A limited memory algorithm for bound constrained optimization. SIAM Journal on Scientific Computing, 16(6), 1190-1208.

Dempster, A. P., Laird, N. M., \& Rubin, D. B. (1977). Maximum likelihood from incomplete data via the emalgorithm. Journal of the Royal Statistical Society, Series B: Statistical Methodology, 39, 1-38.

Dolnicar, S., \& Grün, B. (2007). Assessing analytical robustness in cross-cultural comparisons. International Journal of Tourism, Culture, and Hospitality Research, 1(2), 140-160.

Eilers, P. H. C., \& Marx, B. D. (1996). Flexible smoothing with B-splines and penalties. Statistical Science, 11(2), 89-121.

Fischer, R. (2004). Standardization to account for cross-cultural response bias: a classification of score adjustment procedures and review of research in JCCP. Journal of Cross-Cultural Psychology, 35(3), 263-282.

Fraley, C., \& Raftery, A. E. (2002). Model-based clustering, discriminant analysis and density estimation. Journal of the American Statistical Association, 97(458), 611-631.

Greenleaf, E. A. (1992a). Improving rating-scale measures by detecting and correcting bias components in some response styles. Journal of Marketing Research, 29(2), 176-188.

Greenleaf, E. A. (1992b). Measuring extreme response style. Public Opinion Quarterly, 56(3), 328-351.

Heide, M., \& Gronhaug, K. (1992). The impact of response styles in surveys: a simulation study. Journal of the Market Research Society, 34(3), 215-223.

Hennig, C., \& Liao, T. F. (2013). How to find an appropriate clustering for mixed-type variables with application to socio-economic stratification. Journal of the Royal Statistical Society, Series C, 62(3), 309-333. 
Javaras, K. N., \& Ripley, B. D. (2007). An "unfolding” latent variable model for Likert attitude data: drawing inferences adjusted for response styles. Journal of the American Statistical Association, 102(478), 454 463.

Johnson, T. R. (2003). On the use of heterogeneous thresholds ordinal regression models to account for individual differences in response style. Psychometrika, 68(4), 563-583.

Meng, X.-L., \& Rubin, D. B. (1993). Maximum likelihood estimation via the ECM algorithm: a general framework. Biometrika, 80(2), 267-278.

Paulhus, D. L. (1991). Measurement and control of response bias. In J. P. Robinson, P. R. Shaver, \& L. S. Wrightsman (Eds.), Measures of personality and social psychological attitudes (pp. 17-59). San Diego: Academic.

Ramaswamy, V., Chatterjee, R., \& Cohen, S. H. (1996). Joint segmentation on distinct interdependent bases with categorical data. Journal of Marketing Research, 33(3), 337-350.

Rossi, P. E., Gilula, Z., \& Allenby, G. M. (2001). Overcoming scale usage heterogeneity: a Bayesian hierarchical approach. Journal of the American Statistical Association, 96(453), 20-31.

Scharl, T., Grün, B., \& Leisch, F. (2010). Mixtures of regression models for time-course gene expression data: evaluation of initialization and random effects. Bioinformatics, 26(3), 370-377.

van Rosmalen, J., van Herk, H., \& Groenen, P. J. F. (2010). Identifying response styles: a latent-class bilinear multinomial Logit model. Journal of Marketing Research, 47(1), 157-172.

Wedel, M. (2002). Concomitant variables in finite mixture models. Statistica Neerlandica, 56(3), 362-375.

Wedel, M., \& DeSarbo, W. S. (2002). Market segment derivation and profiling via a finite mixture model framework. Marketing Letters, 13(1), 17-25.

Wedel, M., \& Kamakura, W. (2000). Market segmentation - conceptual and methodological foundations (2nd ed.). Boston: Kluwer.

Wedel, M., Kamakura, W., Arora, N., Bemmaor, A., Chiang, J., Elrod, T., Johnson, R., Lenk, P., Neslin, S., \& Poulsen, C. S. (1999). Discrete and continuous representations of unobserved heterogeneity in choice modeling. Marketing Letters, 10(3), 219-232. 